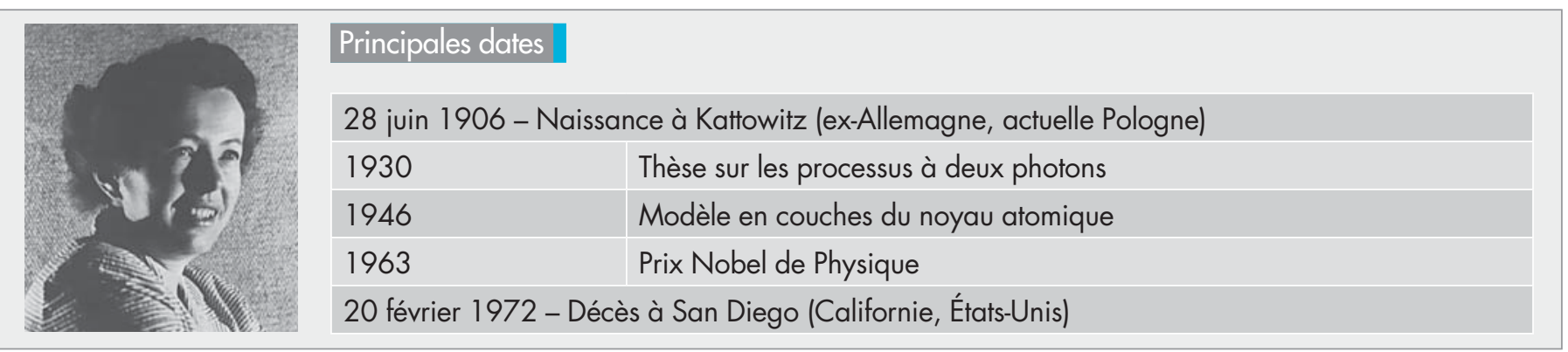

\title{
Maria Goeppert-Mayer
}

\author{
Riad Haidar, haidar@onera.fr
}

Physicienne américaine d'origine allemande, lauréate du prix Nobel pour son modèle en couches du noyau atomique, elle est également connue pour ses travaux théoriques sur l'absorption à deux photons par les atomes - un phénomène que l'invention du laser permettra de vérifier expérimentalement quelques décennies plus tard.

M aria Gerturd Käte Goeppert naît le 28 juin 1906 à Katowice, une ville de l'ancienne province Prusse de Silésie, qui est actuellement la voïrodie de Silésie, localisée au sud de la Pologne. Elle est la fille unique de Maria, née Wolff, et de Friedrich Goeppert, tous deux issus d'anciennes familles allemandes. Son père est, comme cinq générations de Goeppert avant lui, professeur d'université. En 1910, il est nommé à la chaire de pédiatrie de la Georg-August-Universität, et la famille Goeppert s'installe à Göttingen. Friedrich a une influence considérable sur la petite Maria, et fait éclore progressivement sa curiosité scientifique.

Maria fréquente l'école élémentaire publique, puis la Höhere Technische, une école pour jeunes filles aisées. En 1921, elle est admise au FrauenStudium, un institut préparatoire aux études supérieures. Elle obtient son Abitur à Hanovre, à l'âge précoce de 17 ans, et intègre la Georg-August-Universität au printemps 1924. Le département de physique de Göttingen est alors un centre névralgique où se construit la mécanique quantique. Il est dirigé par James Franck et Max Born, qui sont des amis de la famille Goeppert, et sous l'influence desquels Maria se forge un profil de physicienne théorique. Ses facilités en mathématiques font des merveilles, et elle excelle à l'interprétation des spectres de résonnance. Elle décide de préparer une thèse de doctorat sur les processus à deux photons, et la poursuit malgré le chagrin causé par la mort de son père en 1927. Elle la soutient avec succès en 1930, devant un jury composé de trois futurs Prix Nobel (Born, Franck et Windaus). À cette époque, Maria fait la connaissance de Joseph E. Mayer, un étudiant américain qui a rejoint l'équipe de Franck grâce à une bourse Rockefeller, et qui loue une chambre chez les Goeppert. Ils se marient en janvier 1930. Maria adopte le patronyme de GoeppertMayer, et le couple émigre aux États-Unis où Joseph obtient un poste de professeur de chimie à la Johns Hopkins University. Maria s'intègre parfaitement dans la société de Baltimore, et devient citoyenne américaine en 1932. Les Mayer auront deux enfants, Maria Ann et Peter Conrad.

\section{Le départ pour l'Amérique}

Les Mayer resteront neuf ans à Baltimore et, bien qu'elle y enseigne et collabore régulièrement avec son époux et le théoricien Karl $\mathrm{F}$. Herzfeld, avec qui elle publie notamment un article de référence sur la double désintégration bêta, Maria ne parvient pas à obtenir de poste officiel. Outre le sexisme sévère des milieux académiques de l'époque, ce blocage s'explique surtout par l'anti-népotisme strict qui interdit de favoriser l'épouse d'un enseignant-chercheur déjà établi. Elle parvient tout juste à décrocher une position d'assistante dans le département de physique, avec un salaire très faible. Cette activité lui permet cependant de garder un contact avec Göttingen : elle retourne régulièrement en Allemagne et y poursuit sa collaboration avec Born - jusqu'à l'accession au pouvoir de Hitler en 1933, et l'émigration de plusieurs scientifiques allemands vers le reste du monde occidental. Par ailleurs, les Mayer publient Statistical Mechanics, un ouvrage (devenu un classique) de mécanique quantique à l'usage des chimistes.

En 1939, le contrat de Joseph à Johns Hopkins s'achève, et il est recruté à la Columbia University. Maria se voit attribuer un bureau attitré, mais elle n'a toujours pas de poste et pas de salaire. Pugnace et volontaire, elle poursuit ses travaux et nove très vite des liens avec Urey et Fermi. Ce dernier, fraîchement installé aux États-Unis, l'encourage à étudier les éléments de valence des éléments transuraniens. 


\section{Salon des technologies}

en électronique,

mesure,

vision et optique

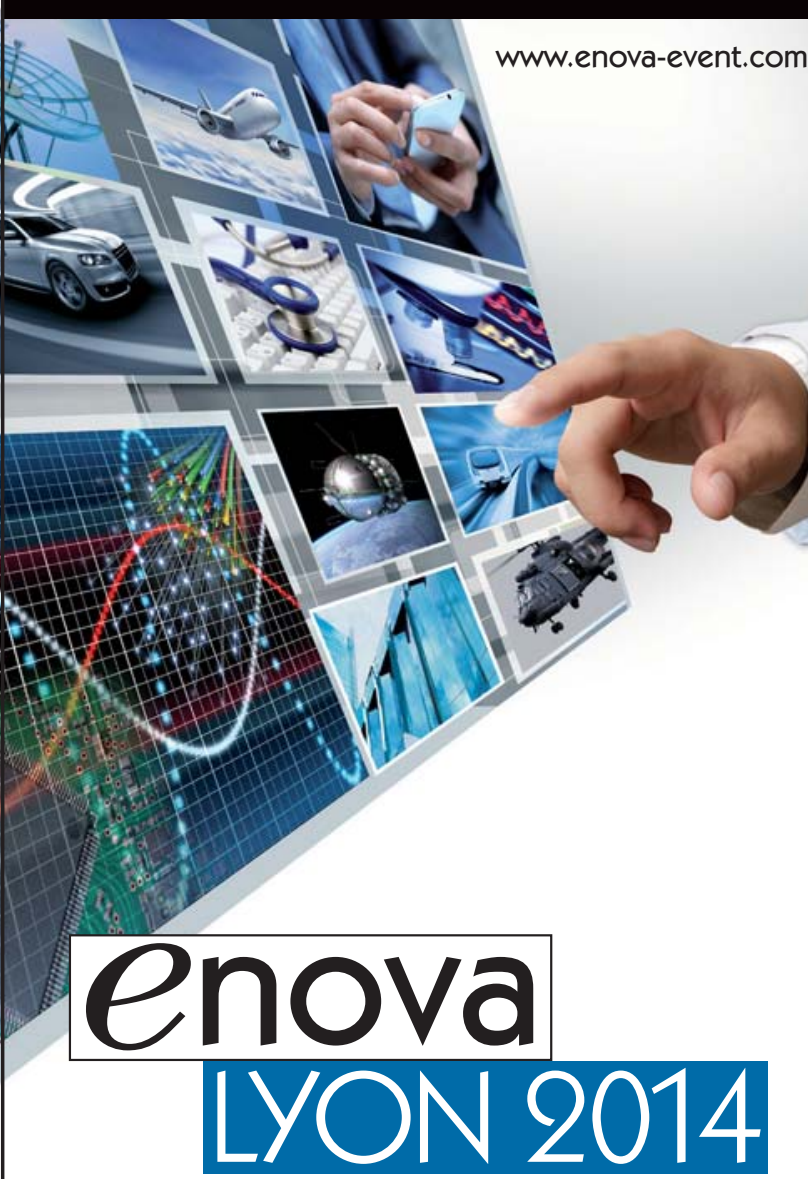

12-13 FÉVRIER

Cité I Centre de congrès

De l'innovation à l'application

\section{Manhattan Project}

En décembre 1941, Maria décroche enfin son premier poste officiel et salarié. Elle enseigne à mi-temps au Sarah Lawrence College et accepte un demi-poste de chercheur à la Columbia University. Au printemps 1942, effort de guerre oblige, elle rejoint le Manhattan Project sur l'enrichissement de l'uranium en son isotope fissile (235U), matière première pour la bombe atomique. En février 1945, Joseph est envoyé dans le Pacifique, tandis que Maria rejoint le groupe de Teller, Bohr et Fermi au Los Alamos Laboratory de New Mexico. Mais cette dispersion familiale ne dure finalement pas, avec la fin précipitée de la guerre contre le Japon.

Au début de l'année 1946, Joseph est nommé professeur à la University of Chicago. Peu de temps après, Maria, qui enseigne bénévolement dans la même université, est recrutée comme chercheur à mi-temps au tout nouvel Argonne National Laboratory. C'est là qu'elle développe, en même temps que les physiciens allemands Wigner et Jensen, le «modèle en couches » du noyau atomique, basé sur le principe d'exclusion de Pauli pour décrire la structure nucléaire en termes de niveaux d'énergie. Elle explique le rôle des «nombres magiques » de Wigner $(2,8,20$, $28,50,82$ et 126), correspondant aux nombres de nucléons qui stabilisent le noyau atomique. Elle signe également, en 1950, un livre avec Jensen, Elementary Theory of Nuclear Shell Structure. En 1963, leurs travaux sont couronnés par le Prix Nobel de Physique. Maria Goeppert-Mayer entre dans la légende en devenant la seconde femme de l'histoire, après Marie Curie, à recevoir cette consécration.

\section{Ultima verba}

En 1959, la University of California contacte Joseph et Maria. C'est une première : Maria obtient un poste de professeur, à temps plein et rémunéré. En réponse, la University of Chicago se décide enfin à proposer un salaire à Maria, mais les Mayer optent pour la Californie et s'installent à La Jolla en septembre 1960.

Peu après, Maria est victime d'un accident vasculaire cérébral qui l'affaiblit considérablement. Elle s'en remet partiellement, et parvient à assurer sa charge d'enseignement pendant encore 12 années. Ses étudiants gardent l'image d'un professeur exigeant, forçant l'écoute en dispensant ses cours d'une voix douce. Droguée au tabac, elle a marqué plusieurs générations d'étudiants en jonglant avec la cigarette et la craie au cours des leçons; I'audience, fascinée par le numéro d'artiste, guettait ses gestes et avait pris I'habitude (mais toujours en vain...) d'attendre que leur professeur s'emmêle avec craie et cigarette, et fume l'une en tentant d'écrire avec l'autre.

En 1971, une attaque cardiaque la précipite dans le coma. Elle décède le 20 février 1972. Elle est enterrée au El Camino Memorial Park de San Diego.

\section{Référence}

R.G. Sachs, Maria Goeppert Mayer, Biographial memoir (1979). 